\title{
Konjunktivistisches Denken oder der Erste Weltkrieg als Lehrstück in Literatur und Wissenschaft
}

\author{
1. Schief gelaufene Interaktionen: zwei \\ historische Großerzählungen um das \\ Balkanproblem
}

Das Verhältnis zwischen fiktionaler und wissenschaftlicher Literatur gilt als geregelt. Fiktionalen Texten wird stoffliche und logische Autonomie zugebilligt, wissenschaftlichen Texten nicht. Letztere haben sich an die Fakten zu halten, was auch und gerade von der Geschichtswissenschaft erwartet wird. Was aber, wenn ein Politikwissenschaftler wie Herfried Münkler Geschichte schreibt, und dann noch die des Ersten Weltkrieges? Auf die von einem Journalisten gestellte Frage, ob der Blick eines Politikwissenschaftlers auf den Ersten Weltkrieg anders ausfällt als der eines Historikers, antwortet Münkler, dass ein Historiker vor allen Dingen Quellen studiere,

Zur Absicht Roths, eine Universitätslaufbahn einzuschlagen, vgl. Sternburg: Joseph Roth, S. 152. Dass Roth, ein Schüler von Brecht, diese Absicht bereits vor Ausbruch des Krieges nicht intensiv verfolgt hat, ist womöglich auf die komplizierte Situation der Wiener Germanistik in diesen Jahren zurückzuführen. Zu Roths Wiener Studienzeit von 1913 bis 1916 s. Bronsen: Joseph Roth, S. 124-153. Zur Situation der Wiener Germanistik in den Vorkriegsjahren s. auch Grabenweger: Germanistik in Wien, S. 50f. sowie Michler: Lessings »Evangelium der Toleranz«, S. 156.
Im Sommer 1914 immatrikulierte sich Joseph Roth an der Wiener Germanistik, wo er gedachte, eine Universitätslaufbahn einzuschlagen. ${ }^{1}$ Der Plan scheiterte am Ausbruch des Ersten Weltkriegs. Statt einer Professur widmete sich Roth fortan dem Versuch, literarisch zu begreifen, was sich ereignet hatte. Vergleichbares unternimmt einhundert Jahre später der Politikwissenschaftler Herfried Münkler. Was beide verbindet, so die These, ist der Zweifel an der "Erzählung der Zwangsläufigkeit des Krieges« (Münkler). Wie detailliert gezeigt wird, liegt dem Konvergieren von Literatur und Wissenschaft eine Öffnung beider für die Kontingenz der Ereignisse und der kontrafaktischen Geschichte zugrunde. 
während ein Politikwissenschaftler sich die Interaktionszusammenhänge anschaut, also wie wer auf wen reagiert und vor allen Dingen, was er daraus lernt. ${ }^{2}$ In diesem Sinne gab Münkler im Jahr 2014 seiner Hoffnung Ausdruck, dass alle Veranstaltungen anlässlich des Kriegsjubiläums dazu führen mögen, dass »Europa innehält und sich auf das in der ersten Hälfte des 20. Jahrhunderts Schiefgelaufene konzentriert, um es im 21. Jahrhundert besser zu machen. $\ll^{3}$ Auf das Schiefgelaufene müsse man schauen, wenn man die Lehren aus der Urkatastrophe ziehen will.

Schief lief es laut Münkler vor allem auf dem Balkan. Vom >Balkan-Problem` nimmt seine Gesamtdarstellung Der große Krieg. Die Welt 1914-1918 ihren Ausgang und bezieht von diesem ihre gegenwartspolitische Relevanz. Münkler, so ließe sich mit einem Seitenblick auf kulturwissenschaftliche Arbeiten der letzten Jahre sagen, bringt den Balkan nicht nur zurück auf die »kognitive Landkarte Europas", sondern räumt ihm auf dieser einen zentralen Platz ein. ${ }^{4}$ Folgt man seiner Darstellung, so sind überhaupt erst nach dem Zerfall Jugoslawiens die Ursprünge des Ersten Weltkriegs wieder deutlich erkennbar geworden. »Er begann auf dem Balkan «, so Münkler in wünschenswerter Klarheit, »und wenn er hier nicht begonnen hätte, wäre er - zumindest zu dieser Zeit - auch im Westen nicht geführt worden «. ${ }^{5}$ Das aber sei erst nach dem Ende des Ost-West-Konflikts wieder ins allgemeine Bewusstsein gedrungen.

Münkler versteht seine Rekonstruktion der Welt zwischen 1914 und 1918 in diesem Sinne auch und nicht zuletzt als ein an die Mitteleuropäer gerichtetes Lehrstück in puncto >nation building` und argumentiert wie folgt: Um die auf dem Balkan immer wieder aufflammenden Staaten- und Bürgerkriege einigermaßen einzuhegen, bedurfte es der permanenten Einwirkung durch eine Ordnungsmacht von außen. Um diese Rolle stritten drei Reiche: Osmanen, Habsburger und das russische Zarenreich. Die Situation war entsprechend unübersichtlich und komplex, richtige Entscheidungen

2 Kapern/Münkler: Münkler: Deutschland hat heute ähnliche ökonomische Rolle wie 1914.

3 Hofmann/Münkler: Der Erste Weltkrieg hat Signalcharakter. Tatsächlich konzentriert sich auch ein Teil der Geschichtswissenschaft vor allem im angelsächsischen Raum auf die Frage: »Was ging schief?« Vgl. hierzu Sked: Der Fall des Hauses Habsburg, S. 40.

4 »Auf der kognitiven Landkarte Europas«, so Gabriella Schubert, »findet der Balkan also keinen oder allerhöchstens einen marginalen Platz. Die mit ihm assoziierten Bilder und Vorstellungen sind finster und furchterregend: Sie präsentieren eine Gegenwelt zum zivilierten Europa, eine Welt des Schreckens, die von Instabilität und unkontrollierbaren Konflikten, von Nationalismus und Historismus sowie von der Unfähigkeit ihrer Bewohner zu dauerhaften und zivilierten Problemlösungen beherrscht werde.« (Schubert: Imaginäre Geographien, S. 44) Wie Schubert betont, haben die Balkankriege und die Ermordung des österreichischen Kronprinzen das negative Bild des Balkans weiter verstärkt (ebd., S. 46).

5 Münkler: Der große Krieg, S. 762. 
schwer zu fällen, und dies umso mehr, als die komplexitätsreduzierende Rolle eines hegemonialen Akteurs im Juli 1914 nicht mehr griff. Trotzdem aber habe es Spielräume für Verhandlungen und gemeinsame Arrangements gegeben, nur seien diese nicht genutzt worden. ${ }^{6}$ Die Politik, d.h. die für verbindliche Entscheidungen zuständige Instanz, erwies sich als eine dem Tempo der modernen Welt nicht gewachsene und versagte. Ein insbesondere für den Raum der Donaumonarchie folgenschweres Versagen, da das habsburgische Vielvölkerreich dafür nicht nur mit seinem Zerfall bezahlte, sondern dieser Zerfall auf dem Territorium des ehemaligen Großreiches außerdem neue Nationalstaaten hinterließ, die ihrerseits allesamt starke nationale Minderheiten aufwiesen. ${ }^{7}$ Kurz: Die Konflikte, die zum Untergang der Donaumonarchie geführt hatten, wiederholten sich auf kleinerem Gebiet.

Im Kontext der Beobachtung dessen, was Münkler auch als >Balkanisierung bezeichnet, d.h. des Zerfalls eines geopolitischen Raums in kleine Einheiten mit Alimentierungsbedarf, stellt sich dem Wissenschaftler die Frage, ob nicht auch die Donaumonarchie politisch hätte überleben können, wenn sie ihre Rettung 1914 nicht im Krieg, sondern in politischen Reformen gesucht hätte. Konkret hätte dies bedeutet: ein höheres Maß an Autonomie für die Nationen und Ethnien des Reichs, wirtschaftliche Integration in den Gesamtraum und die Gewährung einer einheitlichen Außen- und Verteidigungspolitik. Hätte sich das Habsburgerreich so entschieden, wäre es laut Münkler womöglich zum Vorläufer einer mittel- und südosteuropäischen EU geworden. Dass eine solche Entscheidung möglich gewesen wäre, wird von Münkler nicht in Zweifel gezogen. Es hätte anders kommen können, und dass dies nicht geschah, ist zu bedauern. ${ }^{8}$

6 Ebd., S. 47. Bestätigt wird das Bild des persönlichen Versagens einzelner politischer Entscheidungsträger durch detaillierte Darstellungen jüngeren Datums wie Manfried Rauchensteiners Der Erste Weltkrieg und das Ende der Habsburger-Monarchie, hier insbesondere S. 787-795.

7 Vgl. Münkler: Der große Krieg, S. 760.

8 Tatsächlich wurde von dem Hause Habsburg seit dem 17. Jahrhunderts mit einer Mischung aus ökonomischen Anreizen sowie der Präsenz von Polizei und Militär versucht, die Nationalisierungstendenzen auf dem Balkan zu verhindern. Gerade die jüngere Forschung zur Habsburgermonarchie betont die Flexibilität und Reformbereitschaft der Staatsmacht und spricht hinsichtlich des »Paradigma[s] der Rückständigkeit« von einem Revisionsbedarf. Vgl. hierzu Buchen/Rolf: Eliten im Vielvölkerreich, S. 14. Zudem betonen jüngere Arbeiten wie die von Alan Sked, dass es »beinahe keine Hinweise dafür [gebe], daß die Monarchie lebensunfähig war, das heißt, daß eine Mehrheit oder selbst eine bedeutende Minderheit ihrer Bürger ihre Abschaffung gewünscht hätte«. Unterstrichen werden in dieser Lesart der Geschichte die positiven Faktoren, welche die europäischen Reiche vor 1914 insgesamt charakterisieren, nämlich Wirtschaftswachstum und intellektuelle Vitalität (vgl. hierzu Sked: Der Fall des Hauses Habsburg, S. 14 u. 20). Die Münklersche Sicht hingegen wird eher von älteren Arbeiten wie denen des austro-englischen Historikers Robert A. Kann bestätigt. Vgl. hierzu Kann: Werden und Zerfall, S. 20 f. 
In Joseph Roths Radetzkymarsch findet die Münklersche Überzeugung ihre literarische Gestalt. Nachdem das Habsburgerreich untergegangen und der Faschismus in Europa aufgezogen war, beschrieb der jüdische Autor die verschwundene Donaumonarchie im Exil wehmütig als ein Haus mit vielen Türen und Zimmern für viele Arten von Menschen, ein Haus, das man verteilt, gespalten und zertrümmert habe, weshalb er Nationen und Nationalstaaten hasse. Die Verzweiflung angesichts des Untergangs der so charakterisierten »einzigartigen Struktur « der Donaumonarchie deckt sich mit der Position Münklers. ${ }^{9}$ Sie sieht im Recht auf nationale Selbstbestimmung das Verhängnis der europäischen Politik im 20. Jahrhundert und argumentiert, dass dieses Recht eine Fülle von Kriegen und Bürgerkriegen verursachte, da der vormals imperial beherrschte Raum nicht durch Grenzziehungen und Nationalstaaten geordnet werden konnte, ohne neue Minderheiten entstehen zu lassen. ${ }^{10}$ Dabei ist es, so wird an späterer Stelle ausführlicher zu zeigen sein, auch bei Roth das Balkanproblem, an dem diese Position ihre markanteste Gestaltung findet.

Zunächst aber sei festgehalten, dass Roth mit dem Radetzkymarsch, nicht anders als Münkler mit Der Große Krieg, eine historische Großerzählung geschrieben hat. Wie Klaus-Detlef Müller in Anlehnung an Georg Lukács überzeugend dargelegt hat, handelt es sich um einen historischen Roman, und zwar insofern, als in ihm eine krisenhafte Umbruchssituation der Geschichte (eben der Erste Weltkrieg und der Untergang der Habsburgermonarchie) im Erfahrungsbereich mittelmäßiger Protagonisten dargestellt wird, die für sich selbst nur ein abgeleitetes Interesse beanspruchen und von daher nicht von der Gesamtheit des historischen Gegenstands ablenken können. ${ }^{11}$ Schon der Ausgangspunkt, die Schlacht von Solferino, ist laut Müller ein geschichtliches Datum von hoher symptomatischer Bedeutung. Mit ihr erfuhr Österreich 1859 im Feldzug gegen die mit Napoleon III. verbündete italienische Armee eine der beiden entscheidenden Niederlagen. Die Folge war der Verlust der Lombardei, mit dem zum ersten Mal »im Zeichen der Nationalstaatsidee ein Territorium dem universalistischen Herrschaftsgedanken des österreichischen Vielvölkerstaats« entzogen wurde. ${ }^{12}$ Die Schlacht von Solferino, mit der die Romanhandlung einsetzt und die Richtung dieser Handlung vorgegeben wird, gilt demnach als »Beginn

9 Roth: Die Büste des Kaisers, S. 192. Der wie Roth ins Exil getriebene Historiker Kann spricht von der »einzigartigen Struktur « der Donaumonarchie, die sie vom Aufbau der zentralisierten westlichen Nationalstaaten trennte (vgl. Kann: Werden und Zerfall, S. 29).

10 Münkler: Imperien, S. 218. Münkler beruft sich hier auf Eric Hobsbawm.

11 Müller: Joseph Roth, S. 298f.

12 Ebd., S. 301. 
des langen Sterbens der Habsburg-Monarchie «. ${ }^{13}$ Umklammert wird die Handlung von einem zweiten signifikanten Vorgang der Ereignisgeschichte: dem Attentat von Sarajewo, dem wir in Münklers Exposition wieder an zentraler Stelle begegnen. Im Roman stellt sich zwischen beiden Ereignissen »ein Zustand fortschreitender Agonie des Reiches« ein; ein Zustand, der sich nicht in erster Linie den Niederlagen selbst verdankt, sondern vielmehr der Tatsache, dass auf sie unangemessen reagiert wurde. ${ }^{14}$

Mit anderen Worten: Auch Roths Darstellung basiert auf der Annahme, dass es schief gelaufen ist und anders hätte kommen können. Auch in ihr kulminiert die Kette von Fehlentscheidungen im Balkanproblem und entlädt sich in einer Zerstörung, die im Nachhinein zur Hinterfragung der vermeintlichen Zwangsläufigkeit der Ereignisse zwingt. Dies umso mehr, als Roth zu jenen Akteuren der Literaturgeschichte zählt, die das, was Münkler als die Direktionsgewalt des Großen Krieges über das 20. Jahrhundert bezeichnet, unmittelbar zu spüren bekamen. »Mein stärkstes Erlebnis«, so Roth 1932, "war der Krieg und der Untergang meines Vaterlandes, des einzigen, das ich je besessen: der österreichisch-ungarischen Monarchie. $\aleph^{15}$ Die Donaumonarchie, so muss vor dem längeren Blick in den Roman betont werden, war für Roth ein »Ermöglichungsraum autobiographischer Entfaltung «. ${ }^{16}$ In ihm konnte der junge Mann aus Galizien von einer Karriere im Zentrum träumen. Mit dem geopolitischen Raum brachen die Möglichkeiten seiner akademischen Entfaltung zusammen. Und doch, so hat Reinhard Baumgart treffend bemerkt, gehören »der Untergang Europas, seines Europas, und der Aufstieg des Autors Roth in sein

13 Siehe hierzu: Sternburg: Joseph Roth, S. 394. Wie Sternburg festhält, war die Schlacht derart blutig, dass sie den Schweizer Henri Dunant, der dabei war und die Bilder der sterbenden und verwundeten Soldaten nicht vergessen konnte, zur Gründung des Roten Kreuzes veranlasste.

14 Ebd., S. 302.

15 Roth in einem Brief vom 28.10.1932 an Otto Forst de Battaglia. Zit. nach Bronsen: Das literarische Bild der Auflösung, S. 10.

16 Vgl. hierzu: Buchen/Rolf: Eliten im Vielvölkerreich, S. 2. Wie Buchen und Rolf festhalten, war das Reich »kein statisches Gebilde, das es zu erdulden galt«, sondern vielmehr »eine äußerst flexible Bezugsgröße, auf deren Mitgestaltung man Anspruch erhob« (ebd.). Nun gilt dies sicher in erster Linie für eine Elite, zu der Roth (im Unterschied zu dem von Buchen und Rolf eigens besprochenen Robert Musil) nicht gehörte. Doch ließe sich das Forschungskonzept der »imperiale[n] Biographie, die geprägt war von den Strukturen der Reiche, in denen sie lebten, und die sie gleichzeitig zu Meinungs- und Handlungsträgern in den Transformationenprozessen im ausgehenden 19. und beginnenden 20. Jahrhundert machten « (ebd.), auch auf Roth anwenden. Gleiches gilt für die von Buchen und Rolf getroffene Feststellung, dass sich noch in der post-imperialen Phase das Imperium als »Denkfigur und Referenzrahmen, aber auch als biographische Zuschreibung, wenn nicht gar Stigma, als erstaunlich nachhaltig« erwies (ebd., S. 28). 
Erzählreich « zusammen. ${ }^{17}$ Die Position, die Roth in diesem Erzählreich an prominenter Stelle besetzt, ist die des Sachverständigen für Fragen des Zerfalls und des Krieges.

\section{Falsche Heldengeschichten mit fataler Folge}

Im Vorwort zum Vorabdruck des Romans Radetzkymarsch in der »Frankfurter Zeitung « vom 17. April 1932 spricht Roth von dem grausamen Willen der Geschichte, der sein altes Vaterland zertrümmert habe. Es sei fast unmittelbar aus der Operettenvorstellung in das schaurige Theater des Weltkriegs gegangen, und genau dieser Übergang ist das Thema des Romans. Liest man ihn mit den Augen der Politikwissenschaft und konzentriert sich auf schief gelaufene Interaktionen, so fällt zunächst auf, dass Roth den Roman mit einem Fall von Geschichtsfälschung beginnen lässt: der falschen Darstellung der besagten Schlacht von Solferino. Dass die Geschichtsfälschung - trotz besseren Wissens und aller kommunikativen Anstrengungen - zu spät korrigiert wird, erweist sich als eigentlicher Beginn der »Chronik des Untergangs «. ${ }^{18}$ Die Legende, einmal wirkungsvoll verbreitet, lässt sich nicht mehr aus der Welt schaffen. Auf falschen Heldengeschichten und der mit ihnen einsetzenden Verklärung basiert die Darstellung des Rothschen Epochenpanoramas. Die Aufhebung solcher falschen Verklärungen als des »Lebensgrundes einer überlebten Scheinwelt« ist nach Müller das durchgängige Erzählprinzip des Romans. ${ }^{19}$

Bezeichnenderweise ist es ein Lehrwerk, nämlich ein Lesestück für Kinder, in dem sich Hauptmann Trotta, der eigentlich keine Bücher liest, als Protagonist und Held von Solferino wiederfindet. Als Trotta das Stück zufällig bei der Durchsicht der Lesebücher seines Sohnes in die Hände fällt, erfasst ihn ein heftiger Zorn, da das, was in dem Lesestück geschrieben steht, schlicht nicht dem entspricht, was sich wirklich ereignet hatte. Zwar hatte Trotta dem Kaiser in der Schlacht bei Solferino das Leben gerettet, doch war diese Tat eine ganz und gar unspektakuläre, vom Zufall geführte gewesen. Erst die Literarisierung macht Trotta und den Kaiser zu Helden. ${ }^{20}$

17 Vgl. Baumgart: Drei Ansichten, S. 343.

18 Zum Verständnis des Romans Radetzkymarsch als »Chronik des Untergangs« des Habsburgerreichs s. Bronsen: Das literarische Bild der Auflösung, S. 11.

19 Müller: Joseph Roth, S. 305.

20 Eine Literarisierung, die im Roman selbst in Form einer Verflechtung der individuellen Geschichte der Trottas mit dem Leben des Kaisers inszeniert wird, welche wiederum von Beginn 
»Infam « lässt Roth seinen Helden die eigene Heroisierung nennen und ihn mit aller Macht dagegen vorgehen. Aber schon sein erstes Gegenüber, ein Notar, belehrt ihn darüber, dass alle historischen Taten für den Schulgebrauch anders dargestellt werden, da die Kinder Beispiele brauchen, die sie begreifen und sich einprägen können. »Die richtige Wahrheit«, so der Jurist, »erfahren sie dann später! « ${ }^{21}$ Trotta will es bei der falschen Wahrheit nicht bewenden lassen. In Kleistscher Manier lässt Roth das »Martyrium des [...] Ritters der Wahrheit « ablaufen. ${ }^{22}$ Aktion und Reaktion ziehen Kreise. Das Kriegsministerium leitet die Beschwerde an das Kultus- und Unterrichtsministerium weiter. Von dort kommt die Antwort des Ministers: das Lesebuchstück Nummer fünfzehn der autorisierten Lesebücher für österreichische Volks- und Bürgerschulen sei von zwei namhaften Professoren herausgegeben und entspräche den Grundsätzen aller Lesebuchstücke, die da lauten: phantasievolle Darstellung der heroischen Taten unter Anpassung an das Fassungsvermögen der Leser. ${ }^{23}$

Trotta drängt nun auf eine Audienz beim Kaiser. Als er diese endlich bekommt, erfährt er aus höchstem Munde, dass man die Angelegenheit fallen lassen sollte. Zwar sei, so räumt der Kaiser ein, die Sache recht unangenehm, doch kämen weder er noch Trotta dabei schlecht weg. Von daher lautet der kaiserliche Rat: »Lassen S’ die Geschicht'! «"4 Trotta kann

an »im Zeichen falscher Sinngebungen" steht und unlösbare Widersprüche nach sich zieht. Vgl. hierzu: Müller: Joseph Roth, S. 302.

und männlichen Heldenkarrieren in Schulbüchern und zum Prozess der Heroisierung durch Lesebücher für Volksschulen und für die Unterstufen der Gymnasien. Siehe hierzu: Heindl: Helden, Heldinnen und sonstige Idole, S. 145-158. Ein interessanter Aspekt ist zudem die von Günther Ramhardter rekonstruierte Haltung der Mehrheit der Historiker vor und während des Ersten Weltkriegs: fast alle österreichischen Gelehrten erhoben in den Jahren 1914/18 die Forderung nach Konsolidierung der innenpolitischen Verhältnisse Österreich-Ungarns, und dies meinte eine Festigung der Vormachtstellung des deutschen Österreichs. Die Position der akademischen Lehrerschaft bzw. ihr Bemühen um eine Führerstellung der Deutschösterreicher deckte sich mit den Bestrebungen der deutschen bürgerlichen Parteien, die ebenfalls den Ersten Weltkrieg nutzen wollten, um die verworrenen innenpolitischen Verhältnisse zugunsten der Deutschen zu entscheiden. Vgl. hierzu: Ramhardter: Geschichtswissenschaft und Patriotismus, S. 17-30 u. S. 189ff. Zumindest erwähnt werden sollte schließlich, dass Autoren wie Hugo von Hofmannsthal, Robert Musil, Rainer Maria Rilke oder auch Stefan Zweig während des Ersten Weltkriegs im Kriegspressequartier und mithin in einer Institution arbeiteten, deren Aufgabe nach Meinung des Ministerpräsidenten der österreichischen Reichshälfte, Graf Stürgkh, bereits im August 1914 darin bestand, »die Fantasie des Volkes zu befriedigen und so die gute Stimmung zu erhalten«. Vgl. hierzu das Kapitel »Amtlich wird verlautbar« in Rauchensteiner/Broukal: Der Erste Weltkrieg, S. 58f.

Roth: Radetzkymarsch, S. 17. 
es nicht fassen, schließlich ist es eine Lüge. Der Kaiser räumt dies ein, gibt aber zu bedenken, dass viel gelogen werde, er sich jedoch auf seine Minister verlassen können müsse und beendet die Audienz mit den Worten: »Wir wollen's besser machen. Sie sollen sehen! $«^{25}$

Der Kaiser hält sich zunächst nicht an seine Worte. ${ }^{26}$ Anders der Held wider Willen. Roth lässt ihn sofort die Lehre ziehen: Vertrieben aus dem Paradies der einfachen Gläubigkeit an Kaiser und Tugend, Wahrheit und Recht, bittet er um Entlassung aus der Armee und beschließt für seinen Sohn eine Beamten- statt einer Militärkarriere. Im Kopf des Sohnes aber hat sich das Lesestück schon verfangen. Selbst Vater werdend, entscheidet er für seinen Sohn wiederum eine militärische Laufbahn. ${ }^{27}$ Immer wieder kommt dem Sohn, dem jungen Leutnant Trotta, die Heroisierung seines Großvaters zu Hilfe - bis die Operettenvorstellung mit reichlich Alkohol, Frauen und Spiel an ihr Ende kommt. Der Versuch, die Armee zu verlassen, erfolgt zu spät. Das schaurige Theater fängt an.

Dass der Leutnant und die seinen den Umschlag von der Operette zum Schauerstück nicht rechtzeitig erkennen, wird im Roman darauf zurückgeführt, dass sie alle im Frieden geboren und in friedlichen Manövern Offiziere geworden waren. Ihnen allen fehlte die Scharfhörigkeit. »Damals«, so der Erzähler, »wußten sie noch nicht, daß jeder von ihnen, ohne Ausnahme, ein paar Jahre später mit dem Tod zusammentreffen sollte. Damals war keiner unter ihnen scharfhörig genug, das große Räderwerk der verborgenen, großen Mühlen zu vernehmen, die schon den großen Krieg zu mahlen begannen. $\ll^{28}$

Um die verborgenden Mühlen zu vernehmen, bedarf es des Politikverständnisses - und genau daran lässt es der Erzähler im Machtzentrum wie

kerreich, S. 24 und Lindström: Imperial Heimat, S. 385. Dabei ist bezeichnend, dass ausgerechnet die Armee - laut Hilde Spiel »ein Instrument der Völkerverständigung, des friedlichen Ausgleichs der verschiedenen Sprachen, Sitten, Religionen und Wesensarten aller, die ihr dienten « - es ist, in der Roth die Spannungen zwischen den Völkern und ihren Sprachen aufbrechen lässt. Vgl. Spiel: Eine Welt voller Enkel, S. 350. Wie Kann darlegt, waren die Streitkräfte »mit ihrem unbedingten Gehorsam gegenüber dem kaiserlich-königlichen Oberbefehlshaber ungeachtet der immer mehr zersetzenden Wirkung des internen Konflikts ein stark konsolidierender Faktor«. Doch wurden sie mehr und mehr mit dem »besonders nationalbewußten Milieu der unteren Mittelschichten verbunden, aus dem nun die Mehrheit des Offizierskorps stammte « (Kann: Werden und Zerfall, S. 23f.). 
auch im Militär fehlen. »Ich verstehe nichts von Politik! «, entfährt es dem jungen Trotta im Gespräch mit einem Zivilisten..$^{29}$ Nur einer im Roman versteht - der Graf und Reichsratsabgeordnete Chojnicki, eine literarische Figur, die, wie sein Autor, das Habsburgerreich nicht nur vom Zentrum und von der Peripherie her kennt, sondern auch den Blick von außen hat. Er ist der ungläubige und scharfhörige Prophet, der im Roman eben jene Einsicht verkündet, die auch Münkler reformuliert. »Dieses Reich«, so Chojnicki, »muß untergehen. Sobald unser Kaiser die Augen schließt, zerfallen wir in hundert Stücke. Der Balkan wird mächtiger sein als wir. Alle Völker werden ihre dreckigen, kleinen Staaten errichten [...]. « ${ }^{30}$ Die Worte des Grafen vor dem Beginn des Krieges sprechen aus, was Roth bei ihrer Niederschrift Anfang der 1930er Jahre in den Hotelzimmern Mitteleuropas wusste. Das Reich geht unter. Wir alle, so Chojnicki, leben nicht mehr. Zwar habe man noch eine Armee und Beamte, doch zerfalle die Monarchie bei lebendigem Leibe. ${ }^{31}$

Das Zusammentreffen der Trottas mit Chojnicki liest sich wie eine Literarisierung der Münklerschen Charakteristik der Welt von 1914 bis 1918. Münkler spricht von einer »Welt des Übergangs, in der sich Altes und Neues miteinander verbanden, sich vermischten, aber häufig auch bloß unverbunden nebeneinanderstanden «; eine "Zwischenwelt«, die einerseits als Durchbruch in die Moderne wie auch als die Urkatastrophe des 20. Jahrhunderts verstanden werden kann, in der alle Schrecknisse dieser Ära ihren Ursprung hatten. ${ }^{32}$ Mit der Begegnung der Trottas und Chojnicki gewinnt die Spannung von Alt und Neu literarische Gestalt. Chojnicki erklärt dem Bezirkshauptmann - laut Erzähler »ein alter Mann einer alten Zeit ${ }^{33}$ - den Anbruch des Neuen wie folgt: »Die Zeit will uns nicht mehr! Diese Zeit will sich erst selbstständige Nationalstaaten schaffen! Man glaubt nicht mehr an Gott. Die neue Religion ist der Nationalismus. « ${ }^{34}$

Das Zentrum des neuen Nationalismus ist auch im Roman der Balkan. Langsam lässt der Erzähler den Raum bis in das Arbeitszimmer des Bezirkshauptmanns Trotta dringen. Immer mehr verschiedene, schwer verständliche Erlässe und Verfügungen der Statthalterei treffen dort ein. Sie alle sehen eine gelinde Behandlung der nationalen Minoritäten vor. Nationale Minoritäten aber sind für den Beamten nichts anderes als größere 
Gemeinschaften revolutionärer Individuen. ${ }^{35}$ Angesichts ihres Auftauchens setzt der Bezirkshauptmann seine ganze Hoffnung auf die Armee; eine Armee, in der sein eigener Sohn unwillig und leidlich seinen Dienst verrichtet und von der er, der Sohn des unfreiwilligen Helden, wissen müsste, dass sie nie das war, was ihr Glanz suggerierte. Der Schachpartner des Alten und mithin ein Mann strategisch kluger Züge, ist schlauer und erklärt das Dilemma der Donaumonarchie mit den Worten: »Ein junger Offizier unserer Armee kann mit seinem Beruf nicht zufrieden sein, wenn er nachdenkt. Seine Sehnsucht muss der Krieg sein. Er weiß aber, daß der Krieg das Ende der Monarchie ist. ${ }^{36}$

Beide Momente - den Beginn des Krieges und das Ende der Monarchie - führt Roth im Roman wie mit einem Brennglas zusammen. Der Ort der Handlung ist die Grenze, wo man den Krieg regelrecht erwartete. ${ }^{37}$ Im letzten Teil, mitten in eine aus purer Langeweile geplante Feier hinein, platzt die Depesche mit der Nachricht, dass der Thronfolger gerüchteweise in Sarajewo ermordet worden sei. Zunächst als "Zwischenfall« wahrgenommen, von dem man sich die Feierlaune nicht verderben lassen will, dämmert den meisten der Anwesenden bald, dass sich etwas ereignet hat. Die ungarischen Offiziere brechen in Jubel aus und feiern den Mord, »weil es doch allgemein hieß, er (der Thronfolger), sei den slawischen Völkern günstig gesinnt und den Ungarn böse «. ${ }^{38}$ Jelacich, im Roman ein Slowene, gerät darüber in Zorn, weil er die Ungarn ebenso hasst, wie er die Serben verachtet. Seine Liebe gilt allein der Monarchie, doch lässt ihn der Erzähler in diesem Moment auch bedenken, dass seine beiden halbwüchsigen Söhne schon von der »Selbständigkeit aller Südslawen« sprechen. Gleichwohl aber schlägt der Slowene auf den Tisch und bittet die Ungarn darum, ihre Unterhaltung auf Deutsch fortzusetzen. Der Bitte kommt einer der Ungarn gern und mit deutlichen Worten nach: "Wir sind übereingekommen, meine Landsleute und ich, daß wir froh sein können, wann das Schwein hin is! «39

Ebd., S. 278. Wie Kann bereits in den 1960er Jahren festhielt, handelte es sich beim Habsburgerreich streng genommen um einen »Vielvölkerstaat ohne nationale Mehrheit und daher, grundsätzlich betrachtet, auch ohne nationale Minderheiten« (Kann: Werden und Zerfall, S. 21).

36 Roth: Radetzkymarsch, S. 292.

37 Ebd., S. 214. Wie Müller in Anlehnung an Adolf D. Klarmann unterstreicht, wird hier das zu verteidigende Territorium schon vorab zu einem Kriegsgebiet, in dem nationale und soziale Unterdrückung korrespondieren. Die Armee ist hier zugleich gegen den äußeren wie gegen den inneren Feind gerichtet. Die militärische Führung erkennt die Grenzprovinzen schon als Feindesland, das eher besetzt als verwaltet wird. Vgl. hierzu: Müller: Joseph Roth, S. 307. 
Es ist diese Passage des Rothschen Romans, die Münkler zitiert, um seine Version der Geschichte zu entfalten. Dass ein Roman keine verlässliche Quelle ist, spielt für den an schief gelaufenen Interaktionen Interessierten keine Rolle. Im Gegenteil: Die Münklersche Großerzählung gründet auf einer These, die ihrerseits zum Fabulieren regelrecht einlädt, der Annahme nämlich, dass der große Krieg vermeidbar gewesen wäre, hätte man nicht falsche Heldengeschichten erzählt und auf dieser Grundlage politische Fehlentscheidungen getroffen - insbesondere bezüglich der Krisenregion Balkan. Gehen wir also zurück zu Münkler und sehen uns seine Ausführungen näher an, um die Schnittmenge von Literatur und Politikwissenschaft genauer bestimmen zu können.

\section{Kontingenz und Zufall contra Zwangsläufigkeit}

Ganz im Sinne des Romanauftakts, eben der Geschichte vom geschichtsfälschenden Lesestück, attestiert Münkler den europäischen Gesellschaften des 19. Jahrhunderts »einen mentalen Heroisierungsprozess «, welcher im Sommer 1914 kulminierte. »Am 1. August«, so Münkler pointiert, »feierte die heroische Gesellschaft sich selbst. «0 Dass die Feier zum schaurigen Theater gerät, erklärt der Wissenschaftler damit, dass die Politik auf ein zufälliges Ereignis falsch reagiert hat. Das Attentat von Sarajewo - ein Ereignis, mit dem laut Münkler die Kontingenz in die Geschichte zurückkehrt - wird zum eigentlichen Ausgangspunkt einer radikalen Umschreibung der Geschichte. Weit mehr als nur ein Anlass des Krieges, werde das Attentat zu Unrecht von der Suche nach den langfristigen Ursachen des Krieges überschattet. Wenn sich in der Geschichte keine politischen Akteure finden lassen, die den Krieg unter allen Umständen gewollt haben, so müsse man sich von einer Geschichtsschreibung lösen, die den Krieg als überdeterminiert ansieht und sich der Perspektive öffnen, dass das 20. Jahrhundert einen anderen Verlauf genommen hätte, wenn es in Sarajewo nicht zu einer Verkettung unglücklicher Umstände gekommen wäre. Zu Recht habe der amerikanische Sozialwissenschaftler Steven Pinker in seiner Geschichte der Gewalt den Attentäter Gavrilo Princip als die wichtigste Person des 20. Jahrhunderts bezeichnet. ${ }^{41}$

Wenn sich Teile der Wissenschaft, nachgerade der Geschichtswissenschaften, bei der Kriegsursachendebatte dieser Einsicht widersetzen und im 
Attentat bloß den Anlass zu einem ohnehin vorbestimmbaren Prozess sehen, so geschehe dies weniger aus wissenschaftlichen Gründen, als vielmehr aus psychotherapeutischen. Die Vorstellung von der »Wirkmacht des Zufalls« habe nämlich etwas ebenso Verführerisches wie Entsetzliches. ${ }^{42}$ Intuitiv wehre man sich dagegen, dem Zufall einen solchen Einfluss zuzubilligen, da dies hieße, dass der Verlauf der Geschichte völlig unberechenbar und unkontrollierbar sei. Die »Erzählung von der Zwangsläufigkeit des Krieges« sei erträglicher als die von der furchtbaren Macht des Zufalls, weshalb insbesondere deutsche Historiker eine »Großerzählung « geschaffen hätten, in der so viele Wege auf den Krieg zuliefen, dass man ihn buchstäblich nicht mehr verfehlen konnte. ${ }^{43}$

42 Ebd., S. 29. Und an anderer Stelle: »Die Kontingenz der Geschehnisse ist für uns kein Problem, weil von ihr keine grundlegenden Fragen aufgeworfen werden. Aber Ereignisse transzendieren die Normalität oder Banalität des Geschehens; in ihnen stellt sich die Frage nach dem >Wozu und `Warum $\lessdot$. Diese Frage bleibt unbeantwortet, wenn Ereignis und Kontingenz zusammenkommen. Sobald die Vermutung auftaucht, der Zufall habe seine Hand im Spiel gehabt, sind wir bemüht, das Ereignis auf das Niveau eines Geschehnisses herabzustufen. Das beruhigt uns. Wir haben dann wieder ein größeres Vertrauen in den Gang der Dinge. Dementsprechend stellt sich die Frage, ob der Mord von Sarajewo als ein Ereignis oder bloß als ein Geschehnis zu begreifen war. Die Semantik der Herabstufung ist um den Begriff `Anlass herum aufgestellt. Sobald der Mord in einen Anlass verwandelt worden ist, sind wir beruhigt: Es hätte ohnehin so kommen müssen, wie es gekommen ist, selbst wenn es keine Verkettung von Zufällen gegeben hätte.« (ebd., S. 780)

43 Ebd., S. 29. Tatsächlich findet sich bei dem Historiker Kann bereits in den 1960er Jahren eine deutliche Betonung der Rolle des Zufalls bezüglich des Ersten Weltkriegs und der Auflösung der Donaumonarchie: „Es ist kaum möglich, den Anteil anscheinender Zufälle an den stürmischen Ereignissen dieser Zeitspanne auszuschließen " (Kann: Werden und Zerfall des Habsburgerreiches, S. 39). Sicher scheint dem Historiker nur, dass das »letzte Glied in der Kette der Ursächlichkeiten " (ebd.), das zur Auflösung des Kaiserreichs führte, der Erste Weltkrieg war. Auch Rauchensteiner interpretiert die Geschichte eher im Sinne Münklers, wenn er in seiner groß angelegten Studie Der Erste Weltkrieg und das Ende der Habsburger-Monarchie bezüglich des Attentats von Sarajewo schreibt, dass "beim Ablauf der Ereignisse Momente ins Spiel (kamen), die das Attentat noch mehr zum Schicksal werden ließen « (S. 88). Dem schicksalhaften Ereignis folgte laut Rauchensteiner der "Schock « und letztlich die nahezu einhellige Überzeugung in Wien, das »Balkanproblem« ein für alle Mal lösen zu müssen (vgl. ebd., S. 89 u. 90). Ganz anders und durchaus deterministisch die bündige Erklärung Heinrich August Winklers: »Die slawischen Völker hatten seit dem >dualistischen ^Ausgleich mit Ungarn von 1867 darunter zu leiden, daß ihnen die Deutsch-Österreicher und die Magyaren nur mindere Rechte einräumten. Die Erbitterung, die dies erzeugte, untergrub seit dem späten 19. Jahrhundert die Grundlagen der Doppelmonarchie. Die Angst vor der Auflösung des Reiches ließ Österreich nach dem Mord von Sarajewo mit übertriebener Härte auf die Herausforderung durch den großserbischen Nationalismus reagieren und, von der Reichsleitung in Berlin dazu ermutigt, die Politik betreiben, die zum Ersten Weltkrieg führte.« (Winkler: Geschichte des Westens, S. 116) Auch Sked bilanziert: »Es gibt zahllose Meinungsverschiedenheiten im Zusammenhang mit dem Ausbruch des Ersten Weltkrieges. Unumstritten aber ist, daß das österreichisch-ungarische Ultimatum an Serbien mit dem Ziel gestellt wurde, einen Krieg zu entfesseln. Der Krieg ist nicht zufällig ausgebrochen.> (Sked: Der Fall des Hauses Habsburg, S. 299) 
Gegen diese Großerzählung nun schreibt Münkler die seine. Sie beginnt mit der Behauptung, dass der Zufall am 28. Juni 1914 gleich mehrfach seine Hand im Spiel hatte: angefangen von der abgeprallten Bombe, über die Entscheidung des Erzherzogs, das Programm protokollgemäß fortzusetzen, bis hin zu der zweifach geänderten Streckenführung, ohne die Princip keine zweite Chance bekommen hätte. Dem Zufall zu Hilfe kam laut Münkler die Nachlässigkeit und Schlamperei der Verantwortlichen. Gleichwohl aber wäre die Ermordung Franz Ferdinands nicht so folgenreich gewesen, hätten sich mit ihr nicht die Kräfteverhältnisse innerhalb der politischmilitärischen Elite der Doppelmonarchie verändert. Die Falken, d.h. die Befürworter eines Präventivkriegs zur Bändigung der Fliehkräfte innerhalb des Habsburgerreichs bekamen Aufwind und überflügelten jene Fraktion, die einen Ausgleich mit den Slawen und eine Auflösung des Reformstaus favorisierte.

Der eigentliche Fehler der Führung in Wien bestand nach Münkler darin, den Militärschlag zu spät und zudem mit überforderten Streitkräften ausgeführt zu haben. »Österreich-Ungarn war dem Tempo der modernen Welt nicht gewachsen. ${ }^{44}$ Es brach zusammen, weil das Heer den Dienst versagte. Münkler spricht von einem schlechten Führungsstil von Erzherzog Friedrich bis zu den Offizieren, welche den »Krieg als Fortsetzung ihres bisherigen Lebensstils unter erschwerten Bedingungen « verstanden hatten. ${ }^{45}$ In Anlehnung an Rauchensteiner könnte man sagen, dass, weil es in Wien trotz ausreichender Bedenkzeit an kühlen politischen Überlegungen fehlte, "aus einer geplanten kurzen Strafexpedition nach Belgrad ein vier Jahre dauernder Krieg mit 17 Millionen toten Soldaten und Zivilisten « wurde. ${ }^{46}$ Der Glaube der Offiziere, dass der Krieg nichts anderes sei als die Fortsetzung ihres bisherigen Lebensstils unter erschwerten Bedingungen, erwies sich als irrig, und dies im Sachbuch ebenso wie im Roman.

Was begann, war ein Krieg, auf den sich - wechseln wir noch einmal zurück in den Roman - der junge Trotta schon als Siebenjähriger vorbereitet hatte. »Es war sein Krieg «, so träumt der Leutnant, »der Krieg des Enkels. Die Tage und die Helden von Solferino kehrten wieder $«{ }^{47}$ Roth läßt den Traum platzen. Von Heldentaten ist keine Rede. Stattdessen wird nüchtern konstatiert, dass der Krieg der österreichischen Armee mit Militärgerichten begann. »Tagelang hingen die echten und die vermeintlichen Verräter an 
den Bäumen auf den Kirchplätzen, zur Abschreckung der Lebendigen. «48 Und auch das Ende des jungen Trotta selbst war zur Behandlung in Lesebüchern denkbar ungeeignet. »Der Leutnant Trotta starb nicht mit der Waffe, sondern mit zwei Wassereimern in der Hand. «" Zurück in tiefer Trauer lässt der Erzähler den Vater. Die hunderttausend neuen Toten, von denen der Alte am Ende des Romans täglich erfährt, gehen ihn nichts an. »Sein Sohn war tot. Sein Amt beendet. Seine Welt untergegangen. $\aleph^{50}$ Die Romanhandlung schließt.

Dem Schluss aber folgt ein Epilog. Hier schreibt Roth dem sterbenden Kaiser die Worte in den Mund, dass der Krieg eine Sünde sei und man Schluss machen solle. Mehr noch: Auf dem Sterbebett lässt er den Kaiser mit schwacher Stimme sagen: »Wär' ich nur bei Solferino gefallen! ${ }^{51}$ Allein, es ist zu spät und niemand im Roman kann die verwehende Stimme des Kaisers vernehmen. Der Leser jedoch wird durch den Epilog nicht entlassen. ${ }^{22}$ Ihn lädt der abschließende »Bericht aus dem Totenreich « dazu ein, bei den Worten des sterbenden Kaisers aufzuhorchen und sich angesichts des Konjunktivs zu fragen, was gewesen wäre, wenn der Kaiser bei Solferino gefallen wäre. Oder was, wenn der Kaiser gleich eingangs die schulbildende Heroisierung unterbunden hätte? Dann wäre es - vielleicht - nicht schief gelaufen. Vielleicht. In jedem Fall aber hätten sich die Professoren, die die falsche Wahrheit für die Schulbücher verfasst hatten, mit einem Problem auseinandersetzen müssen, das Münkler für ein zu Unrecht verdrängtes hält: das Problem der Kontingenz der Ereignisse.

Wie gesagt, geht Münkler davon aus, dass sowohl Historiker als auch Politikwissenschaftler die Kontingenz des Geschehens in strukturellen Determinanten haben verschwinden lassen. Dass sie dies taten, führt er auf ein »Bedürfnis nach Kontingenzreduktion « zurück, welches die Wissenschaft mit dem Rest der Gesellschaft teile. Mit Roth lässt sich dieser Einschätzung widersprechen. Sein Radetzkymarsch steht für jenen Teil der Gesellschaft, der nicht nur kein Bedürfnis nach Kontingenzreduktion hat, sondern dessen Diskurs geradezu von Kontingenz getragen ist. Die Rede ist von der Literatur - und dies bis hinein in den historischen Ro-

Ebd., S. 386. Eine gesättigte Darstellung der fast »irrationalen Angst vor Spionen« und Kollaborateuren sowie dem Wüten der Stand- und Feldgerichte kurz von dem »Ende der Euphorie« findet sich bei Rauchensteiner: Der Erste Weltkrieg, S. 245ff.

49 Roth: Radetzkymarsch, S. 391.

50 Ebd., S. 394. Mit Rauchensteiner lässt sich die Zahl der Gefallenen präzisieren, wenn er das Ende der k.u.k. Armee unter dem Titel $»$ Vier Millionen Helden« sich anschaulich vor den Augen des Lesers abspielen lässt. Vgl. Rauchensteiner: Der Erste Weltkrieg, S. 972ff.

51 Roth: Radetzkymarsch, S. 401.

52 Scheible: Joseph Roth, S. 73. 
man. Anders formuliert: Was Münkler und Roth verbindet, ist die These der Vermeidbarkeit und der Kontingenz der Ereignisse. ${ }^{53}$ Die historische Darstellung des Politikwissenschaftlers und der historische Roman treffen sich in einem Geschichtsverständnis, das sich der Perspektive öffnet, dass das 20. Jahrhundert einen anderen Verlauf genommen hätte, wenn es in Sarajewo nicht zu einer Verkettung unglücklicher Umstände gekommen wäre. In der »Zwischenwelt « von 1914 hätte es - Roth konnte darüber noch verzweifeln - anders kommen können. ${ }^{54}$ Wir wollen es besser machen, hat der Kaiser im Roman gesagt, es jedoch nicht getan - und am Ende bereut, es nicht getan zu haben.

\section{Möglichkeitsräume, konjunktivisches Denken und systemisches Lernen}

Zur späten Reue aber muss es laut Münkler heute in Mitteleuropa nicht mehr kommen. Erinnern wir uns: Im Kontext einer Beobachtung dessen, was Münkler als > Balkanisierung ‘ bezeichnet, d.h. des Zerfalls eines geopolitischen Raums in kleine Einheiten mit Alimentierungsbedarf, stellt sich für den Politikwissenschaftler die Frage, ob nicht auch die Donaumonarchie hätte überleben können, wenn sie ihre Rettung 1914 nicht im Krieg, sondern in politischen Reformen gesucht hätte. Hätte sich das Habsburgerreich so entschieden, wäre es womöglich zum Vorläufer einer mittel- und südosteuropäischen EU geworden, es hätte keinen Ersten und auch keinen Zweiten Weltkrieg gegeben, »vermutlich auch keinen Nationalsozialismus, keinen Stalinismus, keine bolschewistische Machtergreifung in Petrograd, es wäre ein ganz anderes Jahrhundert gewesen . .5

Ist es aber nicht. Und natürlich kann man fragen, warum kontrafaktische Überlegungen überhaupt von Belang sein sollen. Wen interessiert, was hätte gewesen sein können? Wer operiert im Möglichkeitsraum? Offenbar,

54 Ebd., S. 796.

55 Daneben noch einmal Kann: »Tatsächlich könnte die Monarchie möglicherweise heute noch existieren, wenn der ebenso möglicherweise vermeidbare Erste Weltkrieg nicht ausgebrochen wäre.« (Kann: Werden und Zerfall, S. 40). Das am 17. Oktober 1918 veröffentliche >Völkermanifest‘, so könnte man aus dieser Perspektive sagen, kam einfach zu spät, wobei allerdings zweierlei unterschlagen wird: zum einen die Möglichkeit, dass das Manifest, in dem Kaiser Karl I. seinen Friedenswillen betont, ohne den gerade verlorenen Krieg gar nicht erst geschrieben worden wäre und sich, zum anderen, wesentliche Teile der adressierten Völker zum Zeitpunkt der Veröffentlichung bereits für eine »Zukunft ohne Habsburgermonarchie« entschieden hatten (vgl. Rauchensteiner/Broukal: Der Erste Weltkrieg, S. 237). 
so galt es zu zeigen, bestimmte Segmente der Literatur und der Wissenschaft, und zwar jene, die ihr Augenmerk auf die Geschichte und in ihr wiederum auf (gescheiterte) Interaktionszusammenhänge lenken. ${ }^{56}$ Zur Erfassung und Beschreibung von Wechselwirkungen genügt es ihnen nicht, die tatsächlichen Entscheidungen der Akteure festzuhalten, sondern sie skizzieren auch die Optionen, die den jeweiligen Akteuren zur Verfügung standen. Eine solche Methodik, dies sei noch einmal betont, hat laut Münkler nichts mit retrospektiver Besserwisserei zu tun. Vielmehr liege die Relevanz der Kontingenzthese in der Aussicht auf »systemisches Lernen «. ${ }^{57}$

Was ist damit gemeint? Systemisches Lernen ist ein konstruktivistisches und generatives Lernen, dass auf eine gewollte und zielgerichtete Änderung des Diskurses - in diesem Fall des wissenschaftlichen Diskurses über den Ersten Weltkrieg - abzielt. Eine solche ist laut Münkler nötig, weil die Thesen von Fritz Fischer wie ein politischer Tranquilizer wirkten, der gegenüber den fortbestehenden Konfliktfeldern in Europa unaufmerksam und schläfrig gemacht hat. Der Erste Weltkrieg aber hat laut Münkler keineswegs allein deshalb stattgefunden, weil ihn die politische und militärische Führung des Deutschen Reiches im Juli 1914 gewollt hat. Die jugoslawischen Zerfallskriege haben die Annahme als irrig erwiesen, dass man in Europa solange nicht mit einem Krieg rechnen müsse, solange es kein Regime wie das Wilhelms oder Hitlers gäbe. Die Ereignisse nach 1991 seien eine deutliche Warnung vor dem Irrglauben, die Konstellationen, die in den Ersten Weltkrieg geführt haben, seien überwunden.

Was Münkler wissenschaftlich einholt, wusste Roth aus eigener Anschauung nur zu gut. Der Raum, der seinen »Sinnhorizont « gebildet hatte, war untergegangen, weil die politische Macht wiederholt versagt hatte..$^{58}$ Was blieb, war eine narrative Verarbeitung dieses Versagens. Mit Buchen und Rolf könnte man von einer "autobiographische[n] Stilisierung und [dem] Reflex auf eine Entfremdung von der tristen, unübersichtlichen und zugleich krisengeschüttelten post-imperialen Welt " sprechen. ${ }^{59} \mathrm{In}$ ihr wurde Roth die Fähigkeit, den Standpunkt des Beobachtenden wechseln und im Konjunktiv denken zu können, regelrecht aufgezwungen. Nicht der

Dass es diese auch im Bereich der Geschichtswissenschaften gibt, zeigt die Studie von Sked, der vor allem für die angelsächsische und amerikanische Tradition der habsburgischen Historiographie den »besonderen antifaktischen Charakter« betont. Vgl. Sked: Der Fall des Hauses Habsburg, S. 48.

57 Vgl. hierzu Münkler: Der große Krieg, S. 15 u. 776.

58 Zum Reich als "Sinnhorizont « und zur Wechselwirkung von imperialen Ordnungsmustern und deren Ausdeutung durch die Akteure siehe: Buchen/Rolf: Eliten im Vielvölkerreich, S. 3. 
Verlust an Realitätssinn trägt hier das Kontigenzbewusstsein der Literatur, sondern eine Realität, die Sinnhorizonte untergehen und fortan notwendig Erfahrungen und Erwartungen »im Horizont möglicher Abwandlungen « erscheinen lässt. ${ }^{60}$ Die oft aufgerufenen Worte von der $>$ rückwärtsgewandten Utopie « werden der Erzählerperspektive nicht gerecht. ${ }^{61}$ Vielmehr schrieb Roth, als sich Möglichkeitsräume jenseits der Literatur nicht mehr öffneten, mit dem Radetzkymarsch eine Geschichte, die sich vor dem Hintergrund der nachfolgenden Entwicklungen als vorwärtsgewandte Utopie erweist und sich in den Diskurs über die Grenzen und Möglichkeiten nicht allein der literarischen, sondern auch der geopolitischen Gestaltung einordnen lässt. ${ }^{62}$

Wie ist das zu verstehen? Mit dem Epilog, so konnte gezeigt werden, wird die narrative Schwelle nicht nur zwischen den Erzählwelten überschritten, sondern tritt die Erzählung auch aus ihrem Rahmen heraus und trifft sich mit einem Diskurs, der bis heute disziplin- und systemübergreifend der Frage nachgeht, ob die Urkatastrophe des 20. Jahrhunderts hätte verhindert werden können. Daher sei an dieser Stelle auch jener Position innerhalb der Literaturwissenschaft widersprochen, die Roth und seinen Roman in die Reihe der Verfasser der "nostalgischen Armee-Erinnerungsbücher - von Ginzkey bis Roth, von Roda-Roda bis Lernet-Holenia« einreiht. ${ }^{63}$ Eine solche, den Diskussionsstand der Nachbardisziplinen ausblendende Position verkennt den Stellenwert des Romans nicht nur innerhalb des Kriegs-, sondern auch des Imperiendiskurses. Es gibt bei Roth, dem gebürtigen Juden von der Peripherie, keine »doppelte Verzerrung der Wahrnehmung ", schon gar nicht in " politisch-nationaler Hinsicht «. ${ }^{64}$ Was es statt dessen gibt und was die Texte Roths mit den hier vorgestellten Texten der Wissenschaft verbindet, ist die Wehmut angesichts der verspielten Möglichkeiten und die Hoffnung, dass es die Enkel bei entsprechender Lektüre und einer an ihr geschulten Beobachterperspektive doch noch besser machen können.

Nicht nur Diskurse über die Geschichte werden, so der Kern dieser perspektivischen Einstellung, sondern auch die Geschichte selbst wird gemacht. Ihr Ende ist offen, wenn man den Handlungsverlauf nicht von

60 Zur Definition des Kontingenzbewusstseins vgl. Luhmann: Soziale Systeme, S. 152.

61 Zur `rückwärtsgewandten Utopie`s. Wörsching: Die rückwärts gewandte Utopie; Spiel: Eine Welt voller Enkel, S. 357; Buck u.a.: Von der Weimarer Republik bis 1945, S. 66 und Kindlers Neues Literaturlexikon, S. 352. Sieht man Roths Roman nicht mehr als eine solche, so erscheint er, wie Wörsching konsequent urteilt, als »ein >unpolitischer« Roman, wie ein Neujahrs-Festkonzert konsumierbar, Alt-Österreich...« (ebd., S. 99)

62 Vgl. hierzu Müller: Joseph Roth, S. 319. Zum Ersten Weltkrieg als »Urkatastrophe« des 20. Jahrhunderts s. Winkler: Geschichte des Westens, S. 15.

63 Vgl. hierzu: Strigl: Schneidige Husaren, S. 135.

64 Ebd. 
falschen Heldengeschichten lenken oder ihn nachträglich von Determinismustheorien interpretieren lässt. Schaut man auf die realen Handlungs- und Verhandlungsoptionen, so öffnet sich das, was man als semifiktionalen Raum bezeichnen könnte; ein Raum, in dem konjunktivisches Denken und kontrafaktische Geschichtsschreibung zur Methode, d.h. zum planmäßigen und systematischen Lernverfahren werden. Aus diesem Blickwinkel war der Erste Weltkrieg ein vermeidbares Ereignis, oder, noch deutlicher: »Es war die größte Tragödie Europas, und sie hätte nie geschehen müssen. « ${ }^{65}$ Dies vor Augen, arbeiten beide, Roth und Münkler, mit ihren Narrativen jeweils an einer Änderung des Diskurses und markieren mit der Schnittstelle von Literatur und Wissenschaft den eigentlichen Ort des generativen Lernens. An ihm begegnen wir einer Geschichtsschreibung, die kontrafaktische Überlegungen derart einschließt, dass die Kontingenz des Geschichtlichen innerhalb des faktisch begrenzten Spielraums kenntlich wird. Die dabei mitschwingende Annäherung von Literatur und Wissenschaft an politische Fragen darf nicht als Autonomieverlust gedeutet werden. Das Gegenteil ist der Fall. Wie hier zu zeigen versucht wurde, ist es die spezifische, an das konjunktivistische Moment gekoppelte Macht der Literatur, die als Leistung von anderen Teilbereichen der Gesellschaft zur Überwindung von »Fatalismusfallen, Lernblockaden oder politische[r] Psychotherapie « genutzt wird. ${ }^{66}$ Modell stehen Texte, die den ungeheuerlichen Akt des Herbeischreibens der Geschichte einschließlich seiner Autokorrektur so nachhaltig wie Lehrbuchgeschichten zu gestalten vermögen.

Sked: Der Fall des Hauses Habsburg, S. 44. Der Historiker Sked lässt sich in die Gruppe derjenigen einordnen, die darauf bestehen, dass sich aus dem Fall des Hauses Habsburg (ein Fall ohne Niedergang) Lehren für die Gegenwart ziehen lassen. Gerade weil die europäische Idee die sogenannte `österreichische Staatsidee` heraufbeschwöre, seien alle europäischen Staatsmänner gut beraten, sich über das Schicksal der Habsburger Monarchie Gedanken zu machen. »Wenn sich Europa heute vereinigen will, sollte man wenigstens aus deren Irrtümern lernen können. Das heißt nicht, dass die Geschichte stets ein zuverlässiger Führer ist. Aber wenn man von Staatsmännern erwartet, sich über einen sinnvollen Weg in die Zukunft zu einigen, könnte es kaum schaden und möglicherweise sogar nützen, wenn sie präzise wüssten, was bereits unternommen wurde und worauf das hinauslief.« (ebd., S. 46f.) 


\section{Literaturverzeichnis}

Buck, Theo; Franke, Hans-Peter; Staehle, Ulrich; Wenzelburger, Diemtar (Hgg.): Geschichte der deutschen Literatur. Bd. 5: Von der Weimarer Republik bis 1945. Hgg. Joachim Bark, Dietrich Steilbach. Stuttgart: Klett 1992, S. 63-66.

Böning, Hansjürgen: Joseph Roths »Radetzkymarsch «. München: Fink 1968.

Bronsen, David: Joseph Roth. Eine Biographie. München: dtv 1981 (1974).

Bronsen, David: Das literarische Bild der Auflösung im »Radetzkymarsch «. In: Joseph Roth. Werk und Wirkung. Hg. Bernd M. Kraske. Bonn: Bouvier 1991, S. 9-24.

Buchen, Tim; Malte, Rolf (Hgg.): Eliten im Vielvölkerreich. Imperiale Biographien in Russland und Österreich-Ungarn (1850-1918). Oldenbourg: De Gruyter 2015.

Grabenweger, Elizabeth: Germanistik in Wien. Das Seminar für Deutsche Philologie und seine Privatdozentinnen (1897-1933). Berlin: De Gruyter 2016.

Heindl, Waltraud: Helden, Heldinnen und sonstige Idole. In: Zentren, Peripherien und kollektive Identitäten in Österreich-Ungarn. Hgg. Endre Hárs et. al. Tübingen, Basel: Francke 2006, S. 145-158.

Hofmann, Sarah Judith; Münkler, Herfried: Der Erste Weltkrieg hat Signalcharakter. Interview mit Herfried Münkler. <http://www.dw.com/de/der-erste-weltkrieg-hatsignalcharakter/a-17460936> (Zugriff 14.9.2015).

Jens, Walter: Kindlers Neues Literaturlexikon. Bd. 14. München: Kindler 1988.

Kann, Robert A.: Werden und Zerfall des Habsburgerreiches. Graz, Wien, Köln: Styria 1962. Kapern, Peter; Münkler, Herfried: Münkler: Deutschland hat heute ähnliche ökonomische Rolle wie 1914. Interview mit Herfried Münkler. <http://www.deutschlandfunk.de/erster-weltkrieg-muenkler-deutschland-hat-heute-aehnliche.694.de.html?dram:article_ id=273264> (Zugriff 24.4.2015).

Luhmann, Niklas: Soziale Systeme. Frankfurt/M.: Suhrkamp 1984.

Lindström, Fredrik: Imperial Heimat. Biographies of the "Austrian State Elite" in the Late Habsburg Empire. In: Eliten im Vielvölkerreich. Imperiale Biographien in Russland und Österreich-Ungarn (1850-1918). Hgg. Tim Buchen, Rolf Malte. Oldenbourg: De Gruyter 2015, S. 368-392.

Lukács, Georg: Radetzkymarsch (1939). In: Joseph Roth. Hgg. Daniel Keel, Daniel Kampa. Zürich: Diogenes 2010, S. 432-439.

Michler, Werner: Lessings "Evangelium der Toleranz«. Zu Judentum und Antisemitismus bei Wilhelm Scherer und Erich Schmidt. In: Judentum und Antisemitismus in der österreichischen Literatur. Hgg. Anne Betten, Konstanze Fliedl. Berlin: Erich Schmidt 2002, S. 145-160.

Müller, Klaus Detlef: Joseph Roth: »Radetzkymarsch «. Ein historischer Roman. In: Interpretationen. Romane des 20. Jahrhunderts. Bd 1. Stuttgart: Reclam 1993, S. 298-319.

Münkler, Herfried: Imperien. Die Logik der Weltherrschaft - vom Alten Rom bis zu den Vereinigten Staaten. Berlin: Rowohlt 2005.

Münkler, Herfried: Der große Krieg. Die Welt 1914 bis 1918. Berlin: Rowohlt 2013.

Ramhardter, Günther: Geschichtswissenschaft und Patriotismus. Österreichische Historiker im Weltkrieg 1914-1918. Wien: Verlag für Geschichte und Politik 1973.

Rauchensteiner, Manfried: Der Erste Weltkrieg und das Ende der Habsburger-Monarchie. Wien: Böhlau 2013.

Rauchensteiner, Manfried; Broukal, Josef: Der Erste Weltkrieg und das Ende der HabsburgerMonarchie in aller Kürze. Wien: Böhlau 2015. 
Roth, Joseph: Die Büste des Kaisers. In: ders.: Werke. Bd. 3. Hg. Hermann Kesten. Köln, Berlin: Kiepenheuer \& Witsch 1975 u. 1976.

Roth, Joseph: Radetzkymarsch. München: dtv 2006.

Scheible, Hartmut: Joseph Roth. Stuttgart: Kohlhammer 1971.

Schubert, Gabriella: Imaginäre Geographien der Peripherie. Der Balkan im Spannungsfeld europäischer Paradigmen. In: Zentren, Peripherien und kollektive Identitäten in Österreich-Ungarn. Hgg. Endre Hárs et.al.. Tübingen, Basel: Francke: 2006, S. 42-53.

Sked, Alan: Der Fall des Hauses Habsburg. Der unzeitige Tod eines Kaiserreichs. Berlin: Siedler 1993.

Spiel, Hilde: Eine Welt voller Enkel. Über Joseph Roths »Radetzkymarsch «. In: Romane von gestern - heute gelesen. Bd. 2. Hg. Marcel Reich-Ranicki. Frankfurt/M.: Fischer 1989, S. $350-358$.

Sternburg, Wilhelm von: Joseph Roth. Eine Biographie. Köln: Kiepenheuer \& Witsch 2009.

Strigl, Daniela: Schneidige Husaren, brave Bosniaken, feige Tschechen. Nationale Mythen und Stereotypen in der k.u.k. Armee. In: Zentren, Peripherien und kollektive Identitäten in Österreich-Ungarn. Hgg. Endre Hárs et. al. Tübingen, Basel: Francke 2006, S. 129-143.

Winkler, Heinrich August: Geschichte des Westens. Die Zeit der Weltkriege 1914-1945. München: Beck 2011.

Wörsching, Martha: Die rückwärts gewandte Utopie. Sozialpsychologische Anmerkungen zu Joseph Roths Roman »Radetzkymarsch«. In: Joseph Roth. Hg. Heinz Ludwig Arnold. »Text und Kritik« (1982), S. 90-100. 